\title{
Omega-3 polyunsaturated fatty acids attenuate thrombin formation in human vascular smooth muscle cells
}

\author{
Nikolaos-Taxiarchis Skenteris ${ }^{1,2}$, John Pirault1,2, Silke Thul ${ }^{2}$, Melusine Didelot $^{1}$, Hildur Arnardottir ${ }^{2}$ \\ Patrick Lacolley ${ }^{1}$, Véronique Regnault ${ }^{1}$, Magnus Bäck ${ }^{1,2,3}$
}

'Université de Lorraine, Inserm, DCAC, F-54000 Nancy, France, ${ }^{2}$ Translational Cardiology, Department of Medicine, Karolinska Institutet, Stockholm, Sweden, ${ }^{3}$ Divison of Valvular and Coronary Disease, Karolinska University Hospital, Stockholm, Sweden

Background: Atherosclerosis is a chronic inflammatory disease where phenotypic and functional changes in vascular smooth muscle cells (VSMCs) contribute to some features of this disease, such as thrombosis and vascular calcification ${ }^{1}$. VSMCs provide a significant surface for local thrombin formation in the vascular wall. Thrombin is a potent protease, which in addition to its procoagulant activity exerts proinflammatory effects and participates in the proteolytic metabolism of matrix proteins ${ }^{2}$, including osteopontin (OPN). Although, uncleaved OPN is a strong inhibitor of vascular calcification ${ }^{3}$, the thrombin-cleaved form of OPN (OPN-N half) is associated with carotid-plaque inflammation ${ }^{4}$ and stimulates cardiovascular calcification ${ }^{5}$. Importantly, thrombin generation is increased in VSMCs from spontaneously hypertensive rats ${ }^{6}$ and by cyclic stress ${ }^{7}$ through binding of prothrombin to activated $\alpha_{\nu} \theta_{3}$ integrin. At the same time, omega-3 polyunsaturated fatty acids ( $\mathrm{n}-3$ PUFAs) contribute to decreased inflammation in atherosclerosis ${ }^{8}$ and the thrombotic potential in healthy subjects ${ }^{9}$. Of interest, the recent REDUCE-IT trial reported a $25 \%$ relative risk reduction for cardiovascular events of a high dose of the $n-3$ PUFA eicosapentaenoic acid (EPA) compared with placebo ${ }^{10}$, potentially mediated by the action of an antithrombotic mechanism, which remains to be elucidated.

Hypothesis: The aim of the present study was to investigate the effects of $n-3$ PUFAs on VSMCs' thrombin generation and hence to assess their potential role on the thrombin-mediated OPN cleavage.
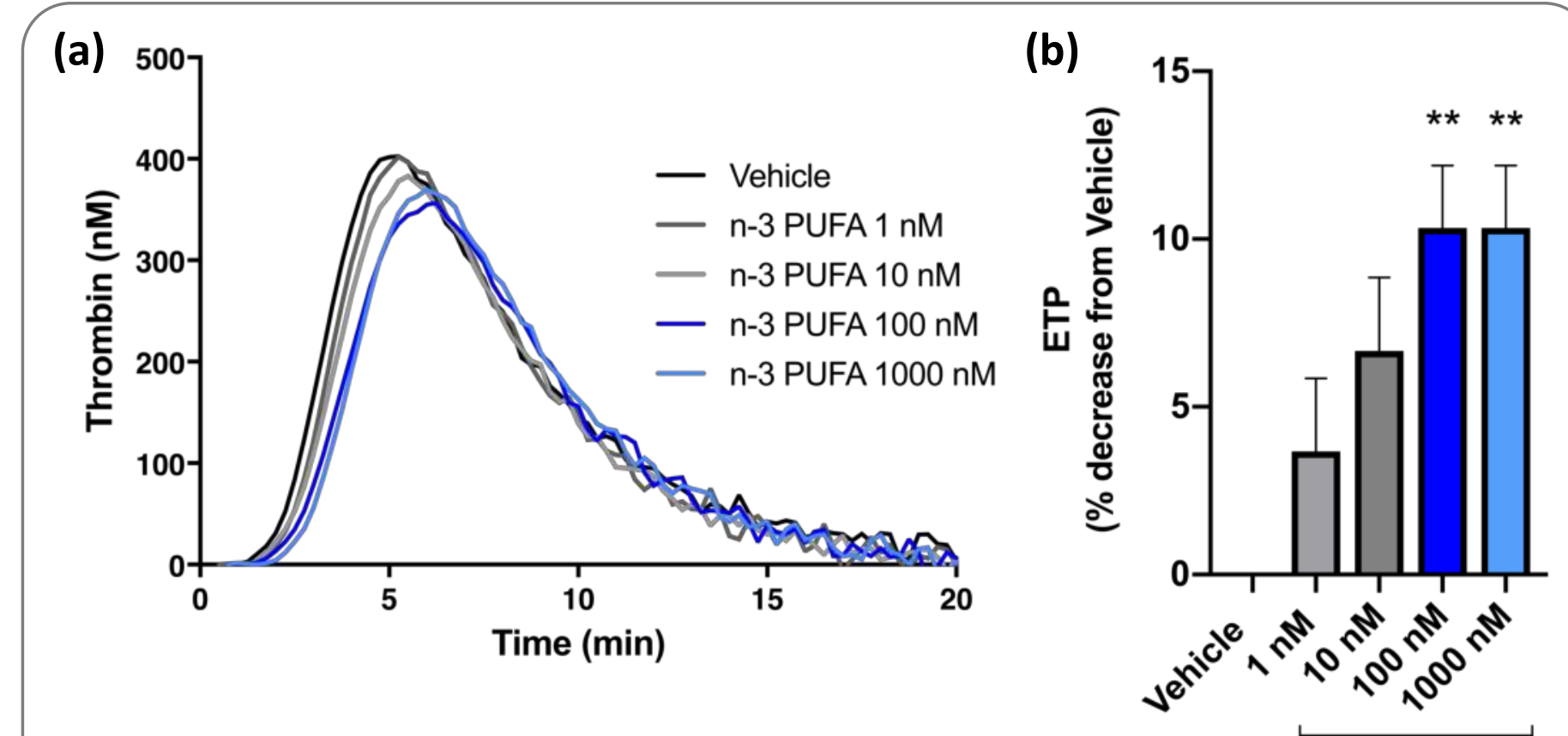

(c)

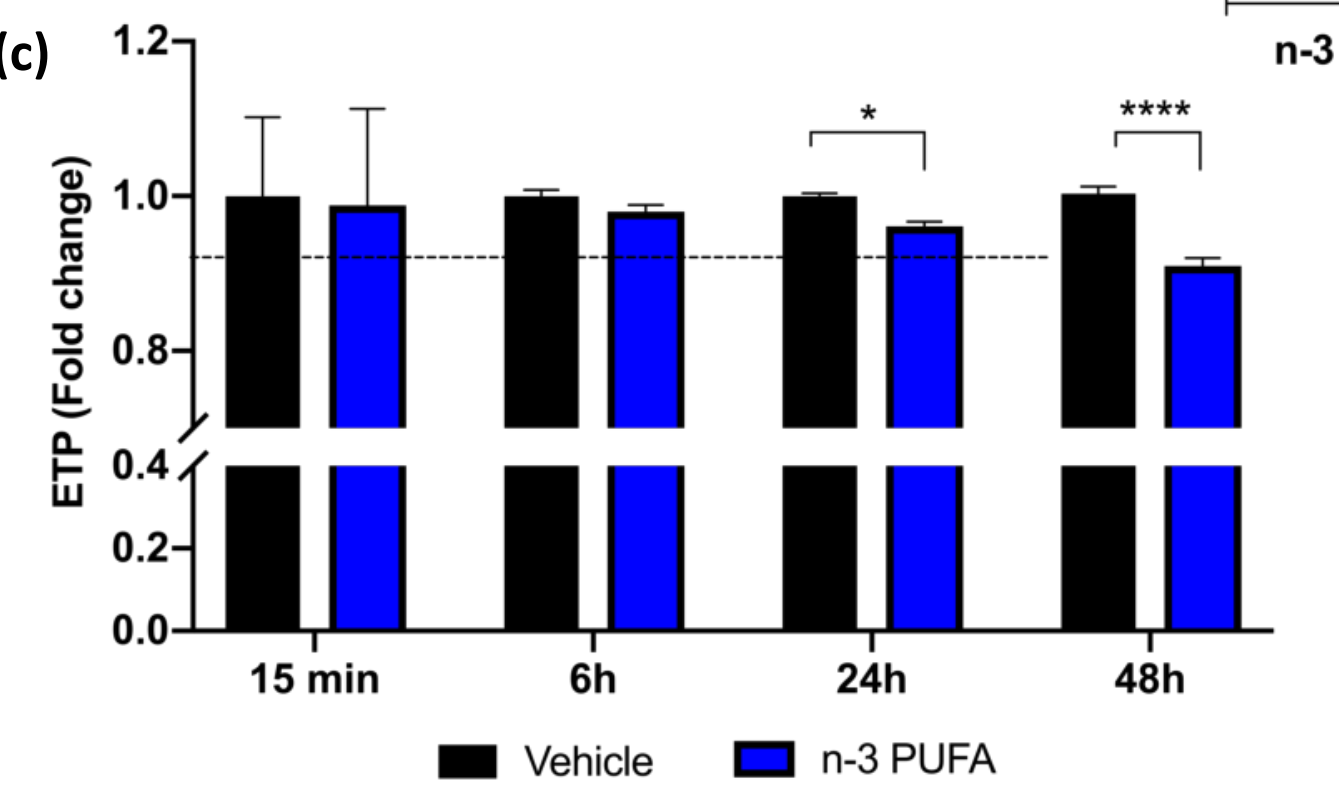

Figure 1. $n-3$ PUFAs reduce thrombin generation at the surface of human vascular smooth muscle cells (VSMCs) (a) Representative thrombin generation curves and (b) \% decrease (from vehicle) in endogenous thrombin potential (ETP) of VSMCs incubated with different concentrations of n-3 PUFA for $48 \mathrm{~h}$ in pooled platelet poor plasma. (c) ETP fold change of VSMCs preincubated with $100 \mathrm{nM} \mathrm{n}-3$ PUFA for $15 \mathrm{~min}, 6 \mathrm{~h}, 24 \mathrm{~h}$ or $48 \mathrm{~h}$ before initiation of thrombin generation. Results are presented as mean \pm SEM from at least 3 experiments in triplicates.
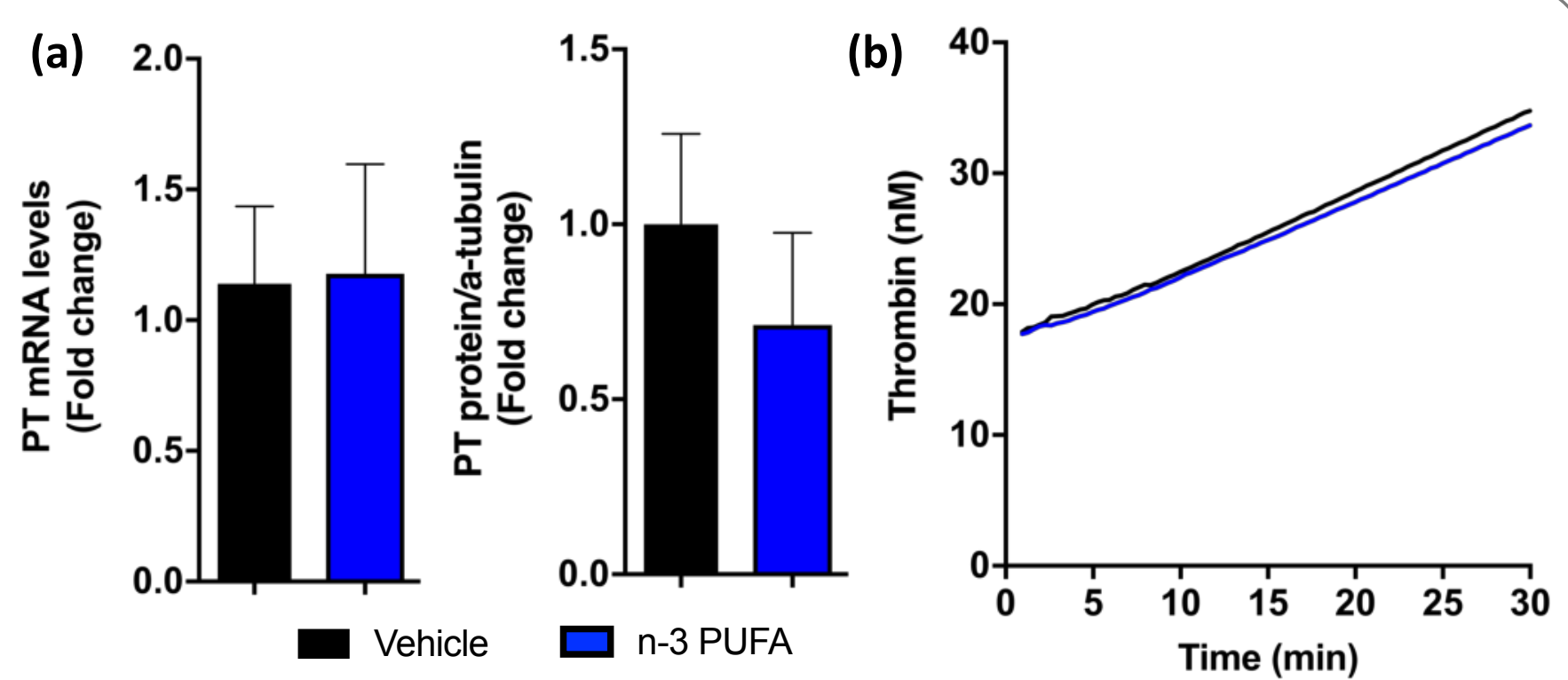

Figure 2. n-3 PUFA-mediated reduction in VSMC thrombin formation is dependent on exogenous prothrombin. (a) Prothrombin mRNA (left) and protein (right) levels from VSMCs incubated with 100nM n-3 PUFA and vehicle for $48 \mathrm{~h}$. (b) Thrombin generation by VSMCs pretreated with vehicle or $100 \mathrm{nM}$ $\mathrm{n}-3$ PUFA for $48 \mathrm{~h}$ in the presence of prothrombin-deficient plasma. Results are mean \pm SEM (a) or representative curve (b) from at least 3 experiments.

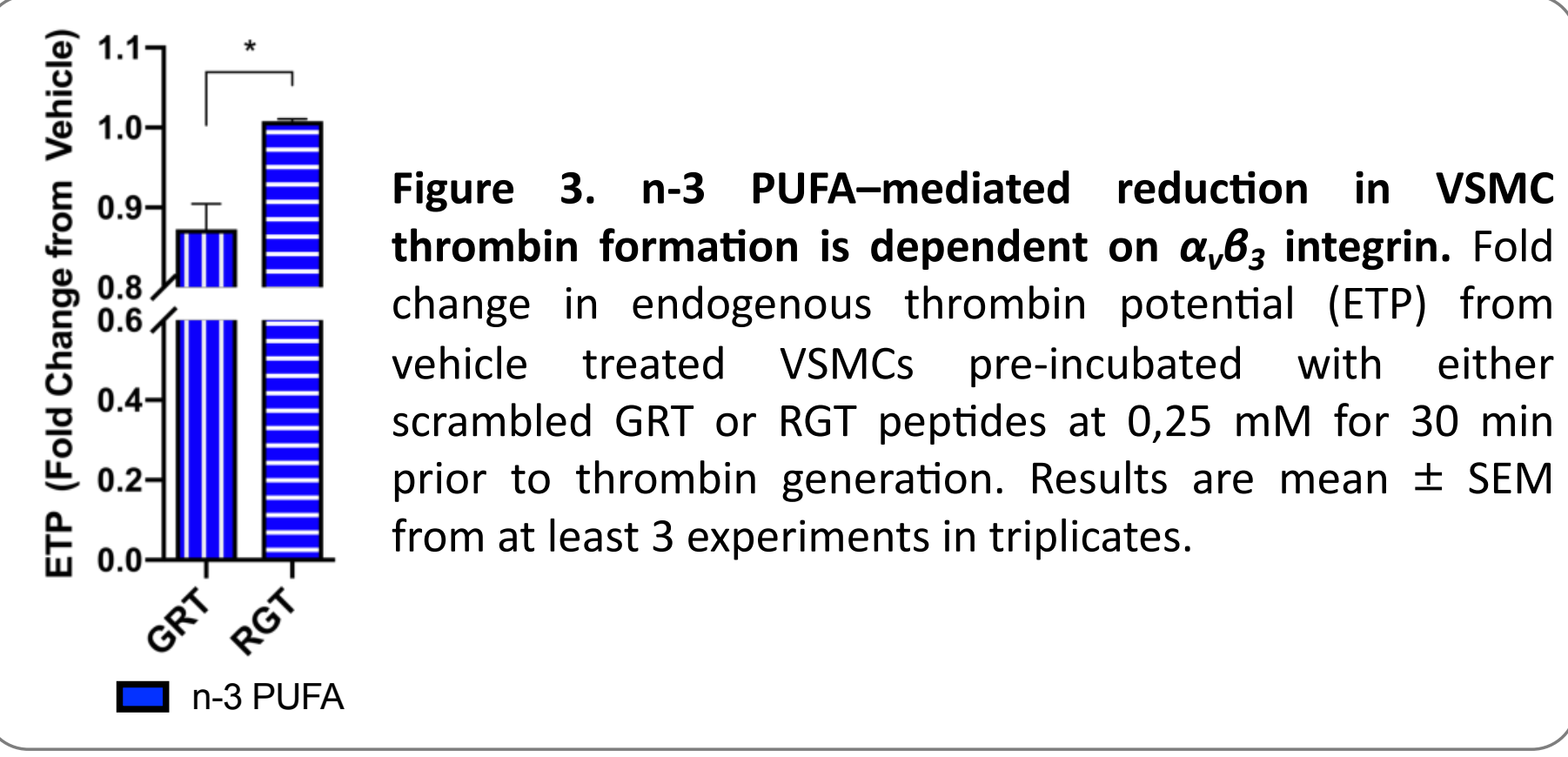

(a)
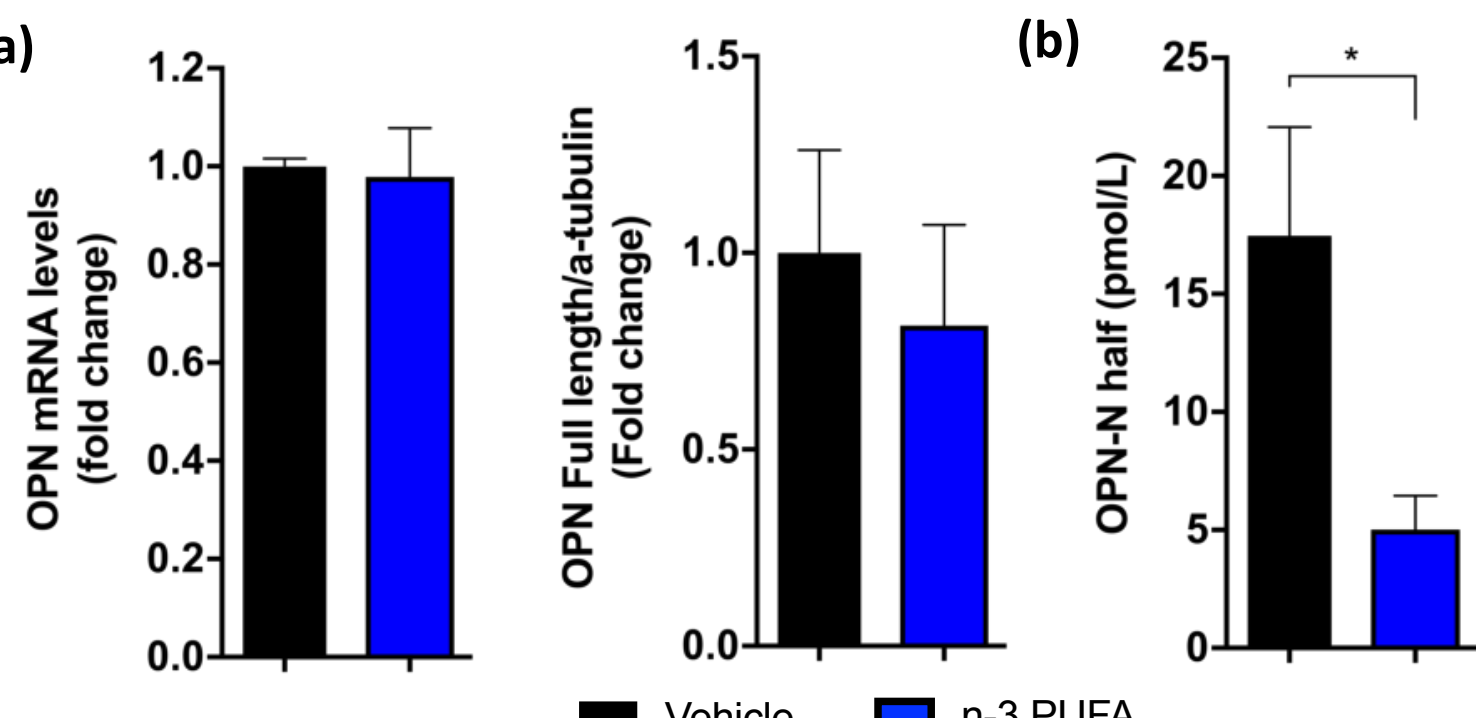

Figure 4. n-3 PUFA reduce thrombin-cleaved Osteopontin $\mathrm{N}$-half. (a) Osteopontin (OPN) mRNA (left) and full-length protein levels (right) from VSMCs incubated with 100nM n-3 PUFA for 48h. (b) Concentration levels of thrombin-cleaved OPN N-half in the supernatants of VSMCs incubated with $100 \mathrm{nM}$ n-3 PUFA for $48 \mathrm{~h}$. Results are presented as mean \pm SEM from at least 3 experiments in triplicates.

\section{Summary - Conclusions}

* n-3 PUFAs reduce thrombin generation in human VSMC

* The n-3 PUFA-mediated reduction of thrombin in human VSMC is dependent on prothrombin and $\alpha_{v} b_{3}$ integrin activation

* n-3 PUFAs reduce the pro-calcifying thrombin-cleaved OPN-N half in human VSMC

* These effects may contribute to the vascular protection of $n-3$ PUFAs

\section{References}

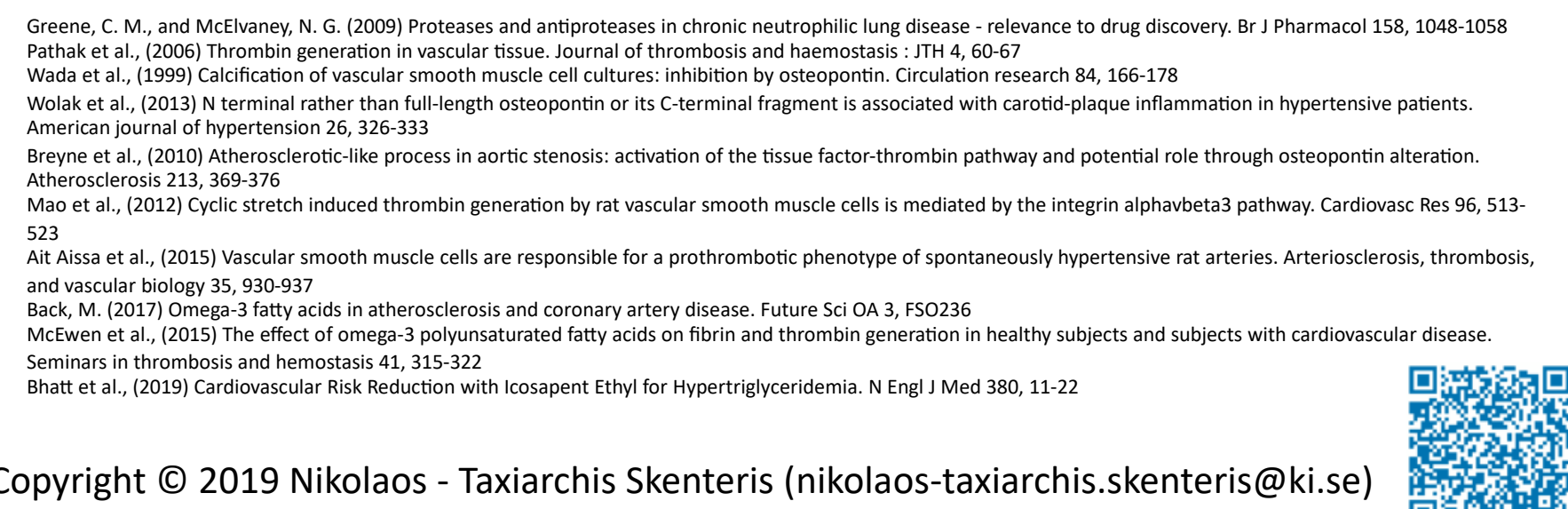

